\title{
Análise das aritméticas de Moore, Kaucher e RDM na solução de problemas de minimização
}

Analysis of Moore, Kaucher and RDM arithmetics in the solution of minimization problems

\author{
D. Maraschin ${ }^{1 *}$; L. M. Tortelli ${ }^{1}$; A. F. Finger' ${ }^{2}$; A. B. Loreto ${ }^{3}$ \\ ${ }^{1}$ CDTec, Universidade Federal de Pelotas, 96075-630, Pelotas, Brasil \\ ${ }^{2}$ Universidade Federal do Pampa, 97546-550, Alegrete, Brasil \\ ${ }^{3}$ Universidade Federal de Santa Maria - Campus Cachoeira do Sul, 96506-310, Cachoeira do Sul, Brasil
}

*dmaraschin@inf.ufpel.edu.br

\begin{abstract}
No trabalho com computação numérica são constatadas diferentes fontes de erros que, quando não são inerentes às incertezas contidas nos dados de entrada do sistema, podem ser ocasionados pelo simples fato de se operar sobre a aritmética de ponto flutuante. Nesse sentido, a aritmética intervalar possui garantias de resultado correto, assim, pode-se oferecer maior confiabilidade nos resultados gerados. É nesse contexto que este trabalho apresentará três aritméticas intervalares: Moore, Kaucher e RDM-IA; sendo apresentadas suas propriedades em termos teóricos, bem como suas formas de representação de valores e operações básicas. Em contrapartida à computação utilizando dados reais, estas três aritméticas intervalares serão testadas utilizando alguns problemas de minimização global, os quais são comumente utilizados no teste de otimização de algoritmos. Todas as computações foram realizadas utilizando a linguagem Python. Ao final, será dado o apontamento sobre qual das aritméticas intervalares demonstra maior confiabilidade e exatidão na busca da solução para os problemas dados no exemplo prático. O objetivo é demonstrar que existem alternativas viáveis no que se refere a problemas ocasionados pelo sistema de ponto flutuante, podendo-se obter resultados confiáveis com garantias de sua qualidade. Além disso, que existem aritméticas intervalares que também são alternativas à clássica de Moore, com abordagens mais recentes, as quais buscam suprimir suas falhas, adicionando ainda mais confiabilidade no resultado.

Palavras-chave: aritmética intervalar, problemas de minimização, computação numérica.
\end{abstract}

In numerical computation, different sources of errors are found which, when they are not inherent to the uncertainties contained in the input data of the system, can be caused by the simple fact of operating on floating-point arithmetic. In this sense, the interval arithmetic has guarantees of correct result. Thus, it can be offer greater reliability in the generated results. In this context, this paper will present three interval arithmetics: Moore, Kaucher and RDM-IA; being presented its properties in theoretical terms, your forms of representation of values and basic operations. In contrast to the computation using real data, these three interval arithmetics will be tested using some global minimization problems, which are commonly used in the algorithm optimization test. All computations were performed using the Python language. At end, it will be given the point about which of the interval arithmetic demonstrates greater reliability and accuracy in the search for the solution to the problems given in the practical example. The objective is demonstrate that there are feasible alternatives with regard to problems caused by the floating point system, and reliable results can be obtained with guarantees of their quality. In addition, show that there are interval arithmetic that are also alternatives to Moore's classic, with more recent approaches, which seek to suppress their flaws, adding even more reliability to the result.

Keywords: interval arithmetic, minimization problems, numerical computation.

\section{INTRODUÇÃO}

A representação numérica em sistemas de computador é limitada considerando o fato de que opera sobre o sistema de ponto flutuante, estando a representação sujeita a erros decorrentes do processo de aproximação para um subconjunto dos números reais, chamados números de máquina.

No processo de resolução de problemas envolvendo quantidades numéricas podem ser constatadas fontes de erros, tais como: propagação de erros contidos nos dados iniciais, arredondamentos e de truncamentos. Considerando que esses erros podem ocorrer em sistemas críticos, onde a exatidão numérica é fundamental, verifica-se uma desvantagem em operar com $049903-1$ 
valores reais. Neste contexto, cabe o emprego de técnicas intervalares, ressaltando-se que em uma resposta intervalar o valor pontual deve estar contido, bem como a resposta exata, normalmente desconhecida. Um valor pontual não possui estimativas de sua incerteza e, mesmo que uma análise de sondagem do erro seja executada, o número resultante é somente uma estimativa do erro que pode estar presente.

A teoria intervalar surgiu com o objetivo de controlar a propagação dos erros numéricos em procedimentos computacionais. Dessa forma, ao utilizar aritmética intervalar em procedimentos numéricos, tem-se controle automático de erros, proporcionado pela aritmética de exatidão máxima e arredondamentos direcionados [12]. Na representação intervalar, um valor real $\mathrm{x}$ corresponde a um intervalo limitado superior e inferiormente, da forma $[x]=[\underline{x}, \bar{x}]$, onde $\mathrm{x}$ deve estar contido.

Embora ainda seja muito utilizada na comunidade científica, a aritmética convencional de Moore et al. (1960) [7] (SIA - do inglês, Standard Interval Arithmetic) possui deficiências que, de modo a contorná-las, outras aritméticas foram sendo definidas. A exemplo disso, existe a aritmética de Kaucher (1980) [4] e, mais recentemente, a aritmética multidimensional RDM [10].

Em vista do que é discutido em trabalhos que apresentam novas aritméticas, o objetivo deste trabalho é apresentar brevemente as aritméticas de Moore, Kaucher e RDM, as respectivas definições teóricas e operações, salientando as deficiências da aritmética de Moore. Como exemplo serão computadas funções que pertencem ao grupo de problemas de otimização global sem restrições nas três aritméticas intervalares retratadas e na aritmética real.

Por fim, espera-se justificar o uso de outra aritmética intervalar alternativamente à convencional de Moore, complementando o que já está sendo trabalhado na literatura e apresentando resultados com base em testes numéricos realizados computacionalmente.

O trabalho está estruturado da seguinte maneira: na seção 2 são apresentados conceitos e propriedades das aritméticas intervalares abordadas nesse trabalho, estando cada uma delas contida em uma subseção específica, começando por Moore, depois RDM-IA e, por fim, Kaucher (1980) [4]. A seção 3 traz o embasamento a respeito dos problemas de minimização abordados no exemplo prático, também os resultados alcançados a parir da computação destes utilizando as aritméticas intervalares abordadas nesse trabalho. Na seção 4 a conclusão faz o fechamento do trabalho com uma discussão geral acerca do trabalho. Por fim, as referências bibliográficas utilizadas.

\section{ARITMÉTICAS INTERVALARES}

A primeira proposta para a solução dos erros numéricos em sistemas computacionais foi concebida por Moore et al. (1960) [7], conhecida como SIA. Esta representa valores de ponto flutuante $\mathrm{x}$ através de intervalos $\left[\underline{x}_{p f}, \bar{x}_{p f}\right]$. Isto proporciona ferramentas robustas de mensuração da qualidade do resultado, agregando garantias aos valores numéricos obtidos, tanto pela sua representatividade como também por fornecer a inexatidão presente no intervalo [12]. Entretanto, SIA contém limitações, como por exemplo: amplitude intervalar excessiva; dependência entre variáveis; problema do lado direito de equações; soluções absurdas, introduzindo entropia negativa ao sistema [2, 14]. Todas estas deficiências somam incertezas às soluções obtidas, contribuindo para gerar um resultado inexato ou incorreto.

Kaucher (1980) [4] visa contornar algumas das limitações presentes em SIA por meio de uma aritmética própria. Uma vez que SIA é incapaz de representar intervalos onde a propriedade $\underline{x} \leq$ $\bar{x}$ não é satisfeita. Desta maneira, Kaucher define a extensão do espaço de representação numérica, em que intervalos do tipo $[\bar{x}, \underline{x}]$, em que $\bar{x} \geq \underline{x}$, são verificados, chamada intervalos impróprios. Kaucher (1980) [4] não apresenta vantagens computacionais quando comparado diretamente à SIA, pois as operações aritméticas definidas para ambas contém a mesma estrutura e operabilidade, tampouco corrige as limitações de SIA diretamente.

A aritmética intervalar multidimensional RDM-IA (do inglês, Relative Distance Measure Interval Arithmetic) [10] apresenta uma abordagem diferente da maioria das aritméticas intervalares, sobretudo das já citadas. Sua representação intervalar para uma quantidade é dada por: $[x]=\left\{x: x=\underline{x}+\alpha_{x}(\bar{x}-\underline{x}), \alpha_{x} \in[0,1]\right\}$, apresentada por meio de um modelo matemático 
como uma função linear. A inserção da chamada variável de incerteza $\alpha$ proporciona melhores resultados, sobretudo quando operações de dependências de variáveis e subtração ocorrem. Entretanto, sua representação no espaço de números intervalares é limitada por IR, não podendo haver intervalos impróprios.

As próximas subseções irão apresentar singularmente cada aritmética abordada neste trabalho, apresentando os detalhes de operabilidade e representação numérica.

\subsection{Aritmética de Moore}

A aritmética intervalar de Moore et al. (2009) [6], Moore (1966) [8] e (1979) [9] utiliza intervalos fechados $[\underline{\mathrm{x}}, \overline{\mathrm{x}}]$ compostos por números reais como elementos básicos. Dada uma função $\mathrm{f}(\mathrm{x})$ de variável real x pertencente a um intervalo $[x]=[\underline{x}, \bar{x}]$ onde $\underline{x}, \bar{x} \in \mathbb{R}$, a imagem de fé dada por:

$$
f([x])=\{y|y=f(x), \underline{x} \leq| x \mid \leq \bar{x}\}
$$

onde esta, em geral, não é representada exatamente, mas é sempre possível determinar um intervalo $[y]=[\underline{y}, \bar{y}]$ tal que $f([x]) \subseteq[y]$, isto é, $\underline{y} \leq f(x) \leq \bar{y}$. Pode-se então definir uma função intervalar $\mathrm{F}$ associada a f pela transformação do intervalo $[\underline{x}, \bar{x}]$ em $[y, \bar{y}]$, isto é $f(x) \subseteq$ $F(x)=[y]$.

Esta função $\mathrm{F}$, chamada extensão intervalar de $\mathrm{f}$, deve ser aquela que possui o mínimo possível de diferença da imagem $f([x])$. O erro obtido no cálculo de $f(x)$ a partir do intervalo $[x]$ é calculado através do diâmetro $w(F([x]))=\bar{y}-\underline{y}$.

A extensão intervalar é definida da seguinte forma:

Definição 1: A função $F: I(\mathbb{R}) \rightarrow I(\mathbb{R})$ é uma extensão intervalar de uma função $f: \mathbb{R} \rightarrow \mathbb{R}$, se para todo $x \in \mathbb{R}, F([x, x])=[f(x), f(x)][13]$.

$\mathrm{Na}$ computação intervalar, pode-se calcular o intervalo solução $[y]=[y, \bar{y}]=$ $f\left(\left[x_{1}\right], \ldots,\left[x_{n}\right]\right)$ através de métodos de aproximação, técnicas de otimização, extensão intervalar e, ainda, por métodos considerados mais sofisticados [5].

Pela definição de Moore, cálculos com intervalos ocorrem sobre conjuntos, ou seja, quando se realiza uma operação sobre dois intervalos, o intervalo resultante é um novo conjunto contendo todos os pares de números das duas séries iniciais operados. Nessa aritmética, aplicando-se o método de extensão intervalar [15], apenas os extremos inferior e exterior do intervalo são considerados.

As operações básicas em Moore seguem as definições:

$$
\begin{aligned}
& {[x]+[y]=[\underline{x}+\underline{y}, \bar{x}+\bar{y}] ;} \\
& {[x]-[y]=[\underline{x}-\bar{y}, \bar{x}-\underline{y}] ;} \\
& {[x] \cdot[y]=[\min (\underline{x} \underline{y}, \underline{x} \bar{y}, \bar{x} \underline{y}, \overline{x y}), \max (\underline{x} \underline{y}, \underline{x} \bar{y}, \bar{x} \underline{y}, \overline{x y})]} \\
& \frac{[x]}{[y]}=[x] \cdot\left[\frac{1}{\underline{y}}, \frac{1}{\bar{y}}\right] \text { se } 0 \notin[y] .
\end{aligned}
$$

As regras mostram que subtração e divisão não são operações inversas da adição e multiplicação, respectivamente, como no caso dos $\mathbb{R}$. Por exemplo:

$$
\begin{gathered}
{[0,1]-[0,1]=[-1,1],} \\
\frac{[1,2]}{[1,2]}=\left[\frac{1}{2}, 2\right] .
\end{gathered}
$$

Essa propriedade é uma das principais diferenças entre aritmética intervalar e real. Outra diferença principal é pelo fato de a regra da distributividade da aritmética real não ser válida em geral. 


\subsection{Aritmética RDM}

$\mathrm{Na}$ aritmética definida por Moore existem algumas inconsistências em cálculos intervalares, como intervalos superestimados, por exemplo. Piegat e Landowski (2012) [10] e Piaget e Marek (2014) [11] desenvolveram a aritmética intervalar RDM dando uma nova perspectiva de representação multidimensional para quantidades intervalares, contornando as problemáticas de SIA.

A abreviatura RDM (do inglês, Relative Distance Measure) significa "Medida da Distância Relativa", sendo considerada multidimensional pelo fato de cada novo parâmetro de incerteza em um sistema aumentar a sua dimensionalidade. Um intervalo $[x]$ é representado como valor RDM da seguinte forma:

onde $\alpha_{x} \in[0,1]$ é uma variável RDM.

$$
[x]=\left\{x: x=\underline{x}+\alpha_{x}(\bar{x}-\underline{x})\right\}
$$

A seguir são apresentadas as operações básicas de aritmética RDM-IA, considerando $[x]$ e $[y]$ intervalos:

$$
\begin{aligned}
& {[x]+[y]=\left\{x+y: x+y=\underline{x}+\alpha_{x}(\bar{x}-\underline{x})+\underline{y}+\alpha_{y}(\bar{y}-\underline{y})\right\}, \alpha_{x}, \alpha_{y} \in[0,1]} \\
& {[x]-[y]=\left\{x-y: x-y=\underline{x}+\alpha_{x}(\bar{x}-\underline{x})-\underline{y}+\alpha_{y}(\bar{y}-\underline{y})\right\}, \alpha_{x}, \alpha_{y} \in[0,1] ;} \\
& {[x] \cdot[y]=\left\{x y: x y=\left[\underline{x}+\alpha_{x}(\bar{x}-\underline{x})\right] \cdot\left[\underline{y}+\alpha_{y}(\bar{y}-\underline{y})\right]\right\}, \alpha_{x}, \alpha_{y} \in[0,1] ;} \\
& {[x] /[y]=\left\{\frac{x}{y}: \frac{x}{y}=\frac{\left[\underline{x}+\alpha_{x}(\bar{x}-\underline{x})\right]}{\left[\underline{y}+\alpha_{y}(\bar{y}-\underline{y})\right]}\right\}, \alpha_{x}, \alpha_{y} \in[0,1], \text { se } 0 \notin[y] .}
\end{aligned}
$$

Para demonstrar a multidimensionalidade da solução utilizando aritmética RDM e a unidimensionalidade utilizando a aritmética de Moore, considera-se operações de adição, subtração, multiplicação e divisão de dois intervalos $[x]=[1,2]$ e $[y]=[3,4]$. Abaixo a solução de cada operação com aritmética de Moore:

$$
\begin{aligned}
& {[x]+[y]=[1,2]+[3,4]=[4,6]} \\
& {[x]-[y]=[1,2]+[3,4]=[-3,-1]} \\
& {[x] \cdot[y]=[1,2] \cdot[3,4]=[3,8]} \\
& {[x] /[y]=[1,2] /[3,4]=[1,2] \cdot\left[\frac{1}{4}, \frac{1}{3}\right]=\left[\frac{1}{4}, \frac{2}{3}\right] .}
\end{aligned}
$$

Para os resultados com aritmética RDM, deve-se escrever os intervalos na notação RDM, ou seja, utilizando as variáveis $\alpha_{\mathrm{a}}$ e $\alpha_{\mathrm{b}}$ :

$$
\begin{aligned}
& {[x]=[1,2]=\left\{x: x=1+\alpha_{x}, \alpha_{x} \in[0,1]\right\}} \\
& {[y]=[3,4]=\left\{y: y=3+\alpha_{y}, \alpha_{y} \in[0,1]\right\} .}
\end{aligned}
$$

Assim, obtém-se as seguintes soluções:

$$
\begin{aligned}
& {[x]+[y]=\left\{x+y: x+y=4+\alpha_{x}+\alpha_{y}, \alpha_{x}, \alpha_{y} \in[0,1]\right\} ;} \\
& {[x]-[y]=\left\{x-y: x-y=-2+\alpha_{x}-\alpha_{y}, \alpha_{x}, \alpha_{y} \in[0,1]\right\} ;} \\
& {[x] \cdot[y]=\left\{x y: x y=3+3 \alpha_{x}+\alpha_{y}+\alpha_{x} \alpha_{y}, \alpha_{x}, \alpha_{y} \in[0,1]\right\} ;} \\
& {[x] /[y]=\left\{x / y: x / y=\left(1+\alpha_{x}\right) /\left(3+\alpha_{y}\right), \alpha_{x}, \alpha_{y} \in[0,1]\right\} .}
\end{aligned}
$$

Para a obtenção dos intervalos solução, as operações são dispostas a seguir:

$$
[x]+[y]=\left[\min _{\alpha_{x}, \alpha_{y} \in[0,1]}\left(4+\alpha_{x}+\alpha_{y}\right), \max _{\alpha_{x}, \alpha_{y} \in[0,1]}\left(4+\alpha_{x}+\alpha_{y}\right)\right]=[4,6]
$$




$$
\begin{aligned}
& {[x]-[y]=\left[\min _{\alpha_{x}, \alpha_{y} \in[0,1]}\left(-2+\alpha_{x}-\alpha_{y}\right), \max _{\alpha_{x}, \alpha_{y} \in[0,1]}\left(-2+\alpha_{x}-\alpha_{y}\right)\right]=[-3,-1] ;} \\
& {[x] \cdot[y]=\left[\min _{\alpha_{x}, \alpha_{y} \in[0,1]}\left(3+3 \alpha_{x}+\alpha_{y}+\alpha_{x} \alpha_{y}\right), \max _{\alpha_{x}, \alpha_{y} \in[0,1]}\left(3+3 \alpha_{x}+\alpha_{y}+\alpha_{x} \alpha_{y}\right)\right]=} \\
& {[3,8] ;} \\
& {[x] /[y]=\left[\min _{\alpha_{x}, \alpha_{y} \in[0,1]}\left[\left(1+\alpha_{x}\right) /\left(3+\alpha_{y}\right)\right], \max _{\alpha_{x}, \alpha_{y} \in[0,1]}\left[\left(1+\alpha_{x}\right) /\left(3+\alpha_{y}\right)\right]\right]=\left[\frac{1}{4}, \frac{2}{3}\right] .}
\end{aligned}
$$

A partir das definições e operações da aritmética RDM, é possível verificar que a inclusão de uma variável RDM faz com que seja minimizado o problema causado quando se utiliza a aritmética de Moore (1966) [8]. A inclusão de parâmetros faz com que a aritmética trabalhe de maneira multidimensional, proporcionando uma melhor representação do intervalo de possíveis valores para uma função.

\subsection{Aritmética de Kaucher}

A Aritmética de Kaucher (1980) [4] surge com a proposta de contornar limitações presentes na aritmética intervalar clássica definida por Moore (1966) [8], a qual é incapaz de representar intervalos onde a propriedade $\underline{x} \leq \bar{x}$ não seja satisfeita. Desta maneira Kaucher (1980) [4] define a extensão do espaço de representação numérica onde intervalos do tipo $[\bar{x}, \underline{x}]$, em que $\bar{x} \geq \underline{x}$, são verificados.

A definição de Kaucher (1980) [4] para intervalos segue os princípios de Moore, onde os cálculos utilizando intervalos ocorrem sobre conjuntos e apenas os limites do intervalo são considerados no cálculo. As operações definidas na extensão intervalar de Kaucher serão apresentadas considerando $[x]=[\underline{x}, \bar{x}]$ e $[y]=[\underline{y}, \bar{y}]$ :

$$
\begin{aligned}
& {[x]+[y]=[\underline{x}+\underline{y}, \bar{x}+\bar{y}] ;} \\
& {[x]-[y]=[\underline{x}-\bar{y}, \bar{x}-\underline{y}]=[x]+[-y], \text { onde }-[y]=[-\bar{y},-\underline{y}]} \\
& {[x] /[y]=[x] \cdot[1 / \bar{y}, 1 / \underline{y}], \text { se } 0 \notin[y] .}
\end{aligned}
$$

A multiplicação definida por Kaucher (1980) [4] é apresentada na Tabela 1, onde: $P=$ $\{[x] \in K \mathbb{R} \mid \underline{x} \geq 0, \bar{x} \geq 0\}, \quad Z=\{[x] \in K \mathbb{R} \mid \underline{x} \leq 0 \leq \bar{x}\}, \quad-P=\{[x] \in K \mathbb{R} \mid-x \in P\}$, dual $Z=\{[\bar{x}] \in K \mathbb{R} \mid$ dual $Z \in Z\}$.

Tabela 1: Extensão intervalar de Kaucher para multiplicação.

\begin{tabular}{ccccc}
\hline$[\mathrm{x}] \cdot[\mathrm{y}]$ & {$[\mathrm{x}] \in P$} & {$[\mathrm{x}] \in Z$} & {$[\mathrm{x}] \in-P$} & {$[\mathrm{x}] \in$ dual $Z$} \\
\hline$[x] \in P$ & {$[\underline{x} \underline{y}, \overline{x y}]$} & {$[\bar{x} \underline{y}, \overline{x y}]$} & {$[\bar{x} \underline{y}, \underline{x} \bar{y}]$} & {$[\underline{x y}, \underline{x} \bar{y}]$} \\
{$[x] \in Z$} & {$[\underline{x} \bar{y}, \overline{x y}]$} & {$\left[\begin{array}{c}\min (\underline{x} \bar{y}, \bar{x} \underline{y}),] \\
\max (\underline{x y}, \overline{x y})]\end{array}\right.$} & {$[\bar{x} \underline{y}, \underline{x y}]$} & 0 \\
{$[x] \in-P$} & {$[\underline{x} \bar{y}, \bar{x} \underline{y}]$} & {$[\underline{x} \bar{y}, \underline{x} \underline{y}]$} & {$[\overline{x y}, \underline{x y}]$} & {$[\overline{x y}, \bar{x} \underline{y}]$} \\
{$[x] \in$ dual $Z$} & {$[\underline{x} \underline{y}, \bar{x} \underline{y}]$} & 0 & {$[\overline{x y}, \bar{x} \underline{y}]$} & {$\left[\begin{array}{c}\max (\underline{x y}, \overline{x y}), \\
\min (\underline{x} \bar{y}, \bar{x} \underline{y})\end{array}\right.$} \\
\hline
\end{tabular}

\section{RESULTADOS E DISCUSSÃO}

A partir dos trabalhos destacados na seção anterior, é possível perceber a possibilidade de aplicação de diferentes aritméticas intervalares como alternativa à computação utilizando valores reais. Além disso, a qualidade dos resultados pode ser estimada, possibilitando uma avaliação da melhor opção na busca pela solução de problemas. 
Com o propósito de aplicar as aritméticas intervalares de Moore, RDM e Kaucher, a presente seção traz um exemplo de aplicação utilizando funções que pertencem ao grupo de problemas de minimização global. Em geral, as técnicas para encontrar a solução global desse tipo de problema enfrentam dificuldades, sendo uma das principais o fato de que o método aplicado pode ficar "preso" em mínimos locais [3]. Em vista disso, problemas de minimização contêm características interessantes para a análise de eficiência, tais como: diferentes complexidades em cada função, convergência sob diferentes abordagens, além da existência de mínimos locais. Dessa forma, estes problemas se fazem interessantes para a análise da capacidade de solução pelas diferentes aritméticas intervalares utilizadas, em comparação às soluções reais.

Entre o conjunto de problemas de minimização classificados como irrestritos, foram escolhidos cinco deles para a computação de suas funções: Ackley, Dixon-Price, Trid, Rastrigin e Sum Squares. As funções que correspondem aos problemas podem ser vistas nas equações (2-6).

$$
\begin{gathered}
\operatorname{ACK}_{n}(x)=20+e^{-\frac{1}{5}} \sqrt{\frac{1}{n} \sum_{i=1}^{n} x_{i}^{2}}-e^{-\frac{1}{n} \sum_{i=1}^{n} x_{i}^{2} \cos \left(2 \pi x_{i}\right)} \\
\operatorname{DIX}_{n}(x)=\left(x_{1}-1\right)^{2}+\sum_{i=2}^{n}\left[i\left(2 x_{i}^{2}-x_{i-1}\right)\right] \\
\operatorname{TRI}_{n}(x)=\sum_{i=1}^{n}\left(x_{i}-1\right)^{2}-\sum_{i=2}^{n} x_{i} x_{i}-1 \\
\operatorname{RAS}_{n}(x)=10 n+\sum_{i=1}^{n}\left[x_{i}^{2}-10 \cos \left(2 \pi x_{i}\right)\right] \\
\operatorname{SUM}_{n}(x)=\sum_{i=1}^{n} i x_{i}^{2}
\end{gathered}
$$

Como parâmetros, foram utilizadas as configurações de computação com 2 e 4 dimensões, 250 iterações e precisão de $\alpha=0.5$, para a aritmética RDM. Os limites que compreendem os domínios de pesquisa para as soluções seguem:

- Ackley: $-15 \leq x_{i} \leq 30, i=1,2, \ldots, n$;

- Dixon-Price: $-10 \leq x_{i} \leq 10, i=1,2, \ldots, n$;

- Trid: $-n^{2} \leq x_{i} \leq n^{2}, i=1,2, \ldots, n$;

- Rastrigin: $-5.12 \leq x_{i} \leq 5.12, i=1,2, \ldots, n$;

- Sum Squares: $-10 \leq x_{i} \leq 10, i=1,2, \ldots, n$.

Seguindo o conceito de utilizar softwares livres e trabalhar com aplicações de código aberto, os testes foram computados na linguagem Python. Atualmente existem implementações da aritmética de Moore, utilizou-se o pacote IntPy [1] para SIA e, para as aritméticas de Kaucher 1980[4] e RDM-IA, serão utilizadas implementações que correspondem a trabalhos de pesquisa dos autores, porém ainda não publicados.

As Tabelas 2, 3, 4 e 5 apresentam os resultados dos problemas de minimização, onde tem-se a solução mínima encontrada para o problema $\left(y_{\min }\right)$, a quantidade de iterações $\left(N_{i t}\right)$ necessárias para encontrar a solução, a dimensão do problema $(\operatorname{Dim})$ e o tempo de execução $\left(t_{c p u}\right)$, em segundos.

O valor $y_{\min }$ presente em cada caso é calculado pela equação (7), a qual calcula a diferença entre a solução real y e o resultado intervalar $\hat{y}$, onde $\varepsilon$ corresponde ao valor residual da operação.

$$
\varepsilon=\mathrm{y}-\hat{y}
$$


Tabela 2: Problemas de minimização global computados com aritmética real.

\begin{tabular}{ccccc}
\hline \multirow{2}{*}{ Problema } & \multicolumn{4}{c}{ Real } \\
\cline { 2 - 5 } & Dim & $\boldsymbol{N}_{\text {it }}$ & $\boldsymbol{y}_{\min }$ & $\boldsymbol{t}_{\text {cpu }}$ \\
\hline Ackley (ACK) & 2 & 4 & $4.4408^{-16}$ & 0.001 \\
Dixon-Price (DIX) & 2 & 214 & 0.1881 & 0.004 \\
Trid (TRI) & 4 & 65 & -56.0 & 0.001 \\
Rastrigin (RAS) & 2 & 3 & 0.0 & 0.007 \\
Sum Squares (SUM) & 2 & 3 & 0.0 & 0.006 \\
\hline
\end{tabular}

Tabela 3: Problemas de minimização global computados com aritmética de Kaucher.

\begin{tabular}{ccccc}
\hline \multirow{2}{*}{ Problema } & \multicolumn{4}{c}{ KaucherPy } \\
\cline { 2 - 5 } & Dim & $\boldsymbol{N}_{i t}$ & $\varepsilon$ & $\boldsymbol{t}_{\text {cpu }}$ \\
\hline Ackley (ACK) & 2 & 10 & $1.7763^{-15}$ & 0.096 \\
Dixon-Price (DIX) & 2 & 214 & -0.000017 & 0.185 \\
Trid (TRI) & 4 & 65 & 0.0 & 0.041 \\
Rastrigin (RAS) & 2 & 11 & 0.0 & 0.127 \\
Sum Squares (SUM) & 2 & 11 & 0.0 & 0.001 \\
\hline
\end{tabular}

Tabela 4: Problemas de minimização global computados com aritmética de Moore, utilizando IntPy.

\begin{tabular}{ccccc}
\hline \multirow{2}{*}{ Problema } & \multicolumn{5}{c}{ IntPy } \\
\cline { 2 - 5 } & $\boldsymbol{D i m}$ & $\boldsymbol{N}_{i t}$ & $\varepsilon$ & $\boldsymbol{t}_{\boldsymbol{c p u}}$ \\
\hline Ackley (ACK) & 2 & 52 & $1.776^{-15}$ & 0.110 \\
Dixon-Price (DIX) & - & - & - & - \\
Trid (TRI) & 4 & 65 & 0.0 & 0.016 \\
Rastrigin (RAS) & 2 & 51 & 0.0 & 0.106 \\
Sum Squares (SUM) & 2 & 51 & 0.0 & 0.003 \\
\hline
\end{tabular}

Tabela 5: Problemas contínuos de otimização global computados com RDM-IA.

\begin{tabular}{ccccc}
\hline \multirow{2}{*}{ Problema } & \multicolumn{4}{c}{ RDM-IA } \\
\cline { 2 - 5 } & Dim & $\boldsymbol{N}_{i t}$ & $\varepsilon$ & $\boldsymbol{t}_{\text {cpu }}$ \\
\hline Ackley (ACK) & 2 & 4 & 0.0 & 20.250 \\
Dixon-Price (DIX) & 2 & 214 & 0.0 & 19.461 \\
Trid (TRI) & 4 & 9 & 0.0 & 3.310 \\
Rastrigin (RAS) & 2 & 3 & 0.0 & 18.496 \\
Sum Squares (SUM) & 2 & 3 & 0.0 & 0.080 \\
\hline
\end{tabular}

Cada função possui propriedades e formas particulares, fazendo com que ofereçam diferentes complexidades na busca pela solução ótima. As soluções reais apresentadas servem de base para a comparação de corretude em primeira instância no comparativo com os resultados intervalares. A partir destes, pode-se observar uma variação na amplitude em que essas soluções foram obtidas quando utilizadas as diferentes aritméticas intervalares. Nesse aspecto, a dimensão se manteve a mesma para todas as aritméticas, respectivamente a cada problema. Já a quantidade de iterações necessárias até o encontro da solução apresenta maior variabilidade.

Particularmente, observando a Tabela 4, pode-se verificar a ineficácia da aritmética de Moore em resolver o problema Dixon-Price. Isso se justifica pelo fato de que SIA não atende a todas as propriedades e, além disso, nem sempre é eficaz na solução de problemas mais complexos, como apontado anteriormente.

Na Tabela 3 foram apresentados os resultados acerca da computação dos problemas utilizando aritmética de Kaucher (1980) [4] onde, para esta, foram obtidas saídas que apresentam valores de acordo com as soluções reais e outras que se afastam minimamente; sendo que os valores apresentados seguem a equação (7).

Os resultados obtidos a partir da computação utilizando RDM-IA, apresentados Tabela 5, mostram que a aritmética foi capaz de encontrar os mínimos globais para todos os problemas abordados. Em vista disso, a aritmética intervalar RDM se apresenta, além de capaz, confiável 
para cálculos numéricos. Observando-se para a coluna correspondente ao tempo de processamento, RDM-IA apresenta os maiores valores. Isso devido ao fato de ocorrer um maior número de comparações ao longo do cálculo até que seja gerado o resultado, visto que cada valor é compreendido por uma função e cada par de valores operados gera um conjunto de valores a ser analisado.

\section{CONCLUSÃO}

Quando se trabalha com computação numérica, um dos fatores de maior importância está na confiabilidade dos resultados obtidos. O que se deseja são resultados com o menor contido possível, alcançando-se resultados mais confiáveis. A aritmética intervalar surgiu com o objetivo principal de realizar o controle automático de erros ocasionados pela aritmética de ponto flutuante, retornando respostas mais exatas.

Diante de diversas aritméticas intervalares que têm sido aplicadas em trabalhos recentes, o presente trabalho teve como objetivo apresentar as vantagens e desvantagens das aritméticas de Moore, RDM [10] e Kaucher (1980) [4]. A aritmética multidimensional RDM surge com o propósito de contornar problemas causados pelo uso da aritmética de Moore, retornando intervalos corretos e com um diâmetro menor. Já a aritmética de Kaucher retrata intervalos impróprios, quando o limite inferior é maior que o superior, o qual não é possível ser representado na aritmética de Moore.

Visto que a aritmética convencional de Moore apresenta diversos problemas, podendo ocasionar em resultados intervalares incorretos ou é incapaz de gerar uma solução, uma alternativa é utilizar a aritmética RDM, a qual tem o intuito de suprir as deficiências da aritmética de Moore. Por outro lado, até o presente momento nada foi demonstrado na literatura que em termos computacionais essa aritmética também se demonstra mais eficiente, tanto em relação à qualidade do resultado quanto em ordem de complexidade.

Com a aritmética de Kaucher (1980) [4] poucos trabalhos são encontrados. Sua diferença em relação a Moore está em suportar intervalos impróprios e para provas matemáticas. Assim, acredita-se que seus resultados possam ser parecidos ou até iguais aos de Moore quanto a complexidade computacional. Mas, nada ainda pode ser afirmado, pois ainda nenhuma análise foi feita.

Como conclusão, podemos afirmar que a aritmética de Moore apresenta algumas deficiências que podem influenciar em resultados incorretos. Por isso, cada vez mais tem sido utilizada aritméticas alternativas que, com os resultados apresentados por alguns trabalhos, bem como aqueles aplicados no presente trabalho, retornam intervalos corretos, podendo solucionar problemas mais complexos com maior confiabilidade.

\section{REFERÊNCIAS BIBLIOGRÁFICAS}

1. Barreto RM, Marcilia AC. Intpy: Um framework intervalar em python. $5^{\circ}$ Encontro Regional de Matemática Aplicada e Computacional. 2008: 31.

2. Dymowa L. Soft computing in economics and finance. Heidelberg: Springer; 2011. 295 p.

3. Hedar A, Ahmed A. Studies on metaheuristics for continuous global optimization problems. 2004.

4. Kaucher E. Interval analysis in the extended interval space IR. Fundamentals of Numerical Computation Computer-Oriented Numerical Analysis. Vienna: Springer; 1980. p. 33-49.

5. Kreinovich V, Lakeyev A, Rohn J, Kahl P. Computational complexity and feasibility of data processing and interval computations. United States: Springer Science \& Business Media; 2013.

6. Moore RE, Kearfott RB, Michael JC. Introduction to interval analysis. Philadelphia: Siam; 2009. 213 p.

7. Moore RE, Wayman S, Yang CT. Interval integrals. [Tech. Rep.] LMSD-703073. Lockheed Aircraft Corporation: Missiles and Space Division, Sunnyvale. California; 1960.

8. Moore RE. Interval analysis. Englewood Cliffs, NJ: Prentice-Hall; 1966. 159 p.

9. Moore RE. Methods and applications of interval analysis. Madison, Wisconsin: Society for Industrial and Applied Mathematics; 1979. 200 p. 
10. Piegat A, Landowski M. Is the conventional interval-arithmetic correct? J Theor Appl Comput Sci. 2012;6(2):27-44.

11. Piegat A, Marek L. Correctness-checking of uncertain-equation solutions on example of the intervalmodal method. Modern approaches in fuzzy sets, intuitionistic fuzzy sets, generalized nets and related topics. 2014; 1:159-170.

12. Ratschek, H, Rokne J. New computer methods for global optimization. Chichester: Horwood; 1988. 229p.

13. Santiago RHN, Bedregal BRC, Acióly BM. Formal aspects of correctness and optimality of interval computations. Formal Aspects Comput. 2006;18(2):231-243.

14. Sevastianov P, Dymova L, Pavel B. A framework for rule-base evidential reasoning in the interval setting applied to diagnosing type 2 diabetes. Expert Syst Appl. 2012;39(4):4190-4200.

15. Sunaga T. Theory of an interval algebra and its application to numerical analysis. Jpn J Ind Appl Math. 2009;26(2):125-143. 